\title{
émulations
}

Luis Martínez - Religion sans rédemption. Contradictions sociales et rêve éveillé en Amérique latine

\section{Guénaëlle de Meeûs}

Émulations - Revue de sciences sociales

2015, «Comptes rendus critiques, En ligne»

Article disponible à l'adresse suivante

https://ojs.uclouvain.be/index.php/emulations/article/view/7043

Pour citer cet article

Guénaëlle de Meeûs, « Luis Martínez - Religion sans rédemption. Contradictions sociales et rêve éveillé en Amérique latine », Émulations, en ligne. Mise en ligne le 10 août 2015.

DOI : 10.14428/emulations.cr.008

Distribution électronique : Université catholique de Louvain (Belgique) : ojs.uclouvain.be

(C) Cet article est mis à disposition selon les termes de la Licence Creative Commons Attribution, Pas d'Utilisation Commerciale 4.0 International. http://creativecommons.org/licenses/by-nc/4.0/

Éditeur : Émulations - Revue de sciences sociales / Presses universitaires de Louvain https://ojs.uclouvain.be/index.php/emulations

ISSN électronique : 1784-5734

UCL PRESSES

UNIVERSITAIRES

DE LOUVAIN 


\section{Luis Martínez Andrade - Religion sans rédemption. Contradictions sociales et rêve éveillé en Amérique latine}

Guénaëlle de Meeûs ${ }^{1}$

Recensé : Luis Martínez Andrade, Religion sans rédemption. Contradictions sociales et rêve éveillé en Amérique latine, Paris, Van Dieren Éditeur, 2015.

Quatre ans après sa parution en espagnol, Religion sans rédemption vient d'être traduit en français. Si la langue change, le ton reste le même : celui de la révolution. Depuis la première publication, l'auteur, Luis Martínez Andrade, diplômé de l'université de Puebla, a soutenu une thèse de doctorat en sociologie à l'EHESS, intitulée "Critique de la modernité et écologie dans la théologie de la libération : la pensée de Leonardo Boff », sous la direction de Michael Löwy.

Luis Martínez Andrade présente ici un essai, ou plutôt un recueil d'essais, « inspiré par la rage et par l'espoir ». Construire une pensée propre à l'Amérique latine en déconstruisant les savoirs occidentaux en est le fil rouge. Depuis l'arrivée de Christophe Colomb sur ses côtes, le continent latino-américain éprouve des difficultés à se dépêtrer des hégémonies politiques, économiques et intellectuelles occidentales. C’est par la prise de conscience et la déconstruction de celles-ci que l'auteur invite à s'en émanciper. À cette fin, l'ouvrage propose une réflexion théorique s'appuyant sur une perspective résolument latino-américaine, guidée par des maîtres à penser de taille : Immanuel Wallerstein, Alejandro Quijano, Enrique Dussel, Leonardo Boff, Ernst Bloch, pour n'en citer que quelques-uns. Son argumentation se décline en cinq chapitres qui ont été écrits de manière indépendante mais peuvent être lus tant pris séparément que comme un ensemble cohérent.

Les chapitres sont regroupés en deux parties. La première retrace la genèse des processus de domination, à la fois culturelle, politique et épistémologique, qui hantent l'Amérique latine depuis plusieurs siècles. Trois concepts majeurs sont mobilisés pour décrire l'établissement de ces hégémonies : le système-monde capitaliste (Wallerstein), la colonialité du pouvoir (Quijano) et l'ego conquiro (Dussel). Le premier chapitre est une critique globale du capitalisme et de l'eurocentrisme toujours ambiants en Amé-

${ }^{1}$ Doctorante au LAAP (Laboratoire d'anthropologie prospective), Université catholique de Louvain. 
rique latine. L'auteur y décrit une colonisation qui n’a pas disparu mais qui a simplement changé de forme. Il introduit ainsi le concept de colonialité du faire et de l'agir. Ce chapitre s'attache en particulier à décrire la mise en place du système-monde vu comme une "formation de la dynamique capitaliste à l'échelle mondiale » qui engendre, depuis son origine, des inégalités. Un système où l'Europe occupe une place centrale depuis laquelle elle impose un imaginaire social à la périphérie. Dans sa présentation du système-monde, Luis Martínez Andrade se penche essentiellement sur le vieux continent qui semble alors être la seule force impérialiste. Néanmoins, on notera que l'auteur parle peu des États-Unis, alors que le sujet aurait mérité d'être abordé. Il poursuit son développement en expliquant que, dans son omnipotence, l'Europe a développé un ego conquiro qui, depuis la colonisation, nie toute altérité des peuples autochtones. Une négation toujours présente actuellement, qu'il est nécessaire de désamorcer pour atteindre l'émancipation. Cette question démontre une certaine complexité de l'Amérique latine d'aujourd'hui. Après cinq cents ans de métissage, comment comprendre qui est exactement l'autochtone? Prolongeant les constats du premier chapitre, le deuxième propose une réflexion sur le centre commercial comme lieu d'expression par excellence de la néocolonialité. Ces malls, toujours plus présents en Amérique latine, sont la matérialisation directe de la consommation de masse, du capitalisme, ainsi que de l'uniformisation des valeurs et des goûts.

Outre ces constats, la deuxième partie du livre propose une ouverture : à la résistance, à l'affranchissement, à la libération et à l'utopie. Elle initie aux différents courants de pensée critiques et libérateurs qui sont présents dans les milieux intellectuels latino-américains. Elle appelle les consciences à s'éveiller au changement. Pour cela, Luis Martínez Andrade se base avant tout sur le principe espérance d'Ernst Bloch qui a été d'une influence notoire sur la philosophie de la libération (Enrique Dussel) et sur la théologie de la libération (Leonardo Boff) ${ }^{2}$. Le religieux est décrit sous deux facettes : celle de l'aliénation et celle de la libération, cette seconde étant privilégiée par l'auteur. Il considère l'espérance et l'utopie véhiculées par le religieux comme des vecteurs d'émancipation pour les classes dominées de la périphérie. La religion se fait libératrice, devient une religion sans rédemption. Les combats menés pour plus d'égalité sociale sont ici associés à ceux qui portent sur la protection de l'environnement. Les dominés et la planète étant les principales victimes du capitalisme, ils se doivent de faire front commun. Religion, environnement, libération : des paroles qui font écho à une actualité récente. En effet, le 16 juin 2015 paraissait la première encyclique entièrement rédigée par le pape François : Laudato si. Révolution et écologie en sont les mots d'ordre, le capitalisme quant à lui y est fustigé. Pas étonnant pour ce premier pape latino-américain qui semble s'inscrire chaque jour un peu plus dans l'héritage de la théologie de

2 À ce sujet, voyez Luis Martínez Andrade, Écologie et Libération. Critique de la modernité dans la théologie de la libération, Paris, Van Dieren Éditeur, 2015. 
la libération. Monde académique, société civile, hautes instances religieuses: nombreux sont ceux qui se préoccupent des grands bouleversements vécus par notre époque et s'accordent pour affirmer qu'une sortie des hégémonies ambiantes et un changement de paradigme semblent devenir incontournables. En ligne de mire, la lutte pour plus d'égalité sociale et la question environnementale sont mises au cœur des débats.

Avec Religion sans rédemption, Luis Martínez Andrade réussit le pari d'une synthèse théorique dense et originale proposant des pistes intéressantes. En combinant un travail de recherche fouillé et des positionnements éthiques et politiques assumés, il démontre que la recherche scientifique n'est pas nécessairement incompatible avec une certaine forme d'engagement.

L'ouvrage amène des propositions en faveur d'un changement radical des pensées dont la nécessité ne peut plus être niée dans la conjoncture actuelle. L'histoire a longtemps transmis une version universaliste et univoque de la colonisation: celle des vainqueurs, celle de l'homme blanc, celle de l'Europe. Déconstruire ces hégémonies s'avère aujourd'hui crucial pour l'Amérique latine, mais pas uniquement. À l'heure de la globalisation, cet impératif ne se limite pas à un seul continent; il doit avoir une visée plus large. On peut ainsi reprocher à certains penseurs radicaux latino-américains, dont l'auteur du présent ouvrage, de présenter une vision de l'Europe dont le principal défaut est d'être trop manichéenne. Tout au long de ce livre, le vieux continent apparaît souvent comme un nid de bourreaux impérialistes opposé aux opprimés de la périphérie. Une vision sans doute trop dualiste qui ne rend pas compte de la complexité d'une Europe de plus en plus plurielle et dont une grande part de la population est elle-même victime de l'hégémonie capitaliste. Sans nier le rôle dévastateur qu'a joué l'Europe, il importe néanmoins de faire la part des choses et de ne pas la présenter comme un bloc univoque tout puissant. La lutte pour une libération de la pensée, de la politique et de la culture est en marche et doit se perpétuer. Mais pour mener ce combat à bien, il nous semble qu'introduire davantage de nuances dans les débats ne serait que bénéfique. Une nuance qui éviterait d'enfermer l'un ou l'autre pôle dans sa position, une nuance qui éviterait la tentation des manichéismes, une nuance qui permettrait ainsi le rassemblement des forces et éviterait les désunions, ultimement néfastes aux luttes et aux objectifs qu'elles poursuivent. 